\title{
Comments on "A new method of analysis of the near and far fields of paraboloidal reflectors"
}

\section{Bach, Henning}

Published in:

I E E E Transactions on Antennas and Propagation

Publication date:

1985

Document Version

Publisher's PDF, also known as Version of record

Link back to DTU Orbit

Citation (APA):

Bach, H. (1985). Comments on "A new method of analysis of the near and far fields of paraboloidal reflectors". I E E E Transactions on Antennas and Propagation, 33(1), 116-118.

\section{General rights}

Copyright and moral rights for the publications made accessible in the public portal are retained by the authors and/or other copyright owners and it is a condition of accessing publications that users recognise and abide by the legal requirements associated with these rights.

- Users may download and print one copy of any publication from the public portal for the purpose of private study or research.

- You may not further distribute the material or use it for any profit-making activity or commercial gain

- You may freely distribute the URL identifying the publication in the public portal

If you believe that this document breaches copyright please contact us providing details, and we will remove access to the work immediately and investigate your claim 
are then readily shown to be the same as those for the convex ellipsoidal system with $\alpha$ and $\beta$ interchanged.

\section{REFERENCES}

[1] H. Tanaka and M. Mizusawa, "Elimination of cross polarization in offset dual-reflector antennas," Elec. Commun. Japan, vol. 58, pp. $71-78,1975$.

[2] Y. Mizuguch, M. Akagawa, and H. Yokoi, "Offset dual reflector antenna," Digest 1976 AP-S Int. Symp. Antennas Propagat., Oct 1976.

[3] C. Dragone, "Offset multireflector antennas with perfect pattern symmetry and polarization discrimination," Bell Syst. Tech. J, yol. 57, pp. 2663-2684, Sept. 1978.

[4] Personal communication to Mr. Carlyle J. Sletten.

\section{Comments on "A New Method of Analysis of the Near and Far Fields of Paraboloidal Reflectors"}

\section{HENNING BACH}

In the above paper ${ }^{1}$ the radiated far field from a reflector antenna is predicted by a "new" method, that determines the far field by a spherical wave expansion of the near field on a sphere enclosing the antenna, once the near field has been found using the geometrical theory of diffraction (GTD). However, this technique of combining a spherical near-field (SNF) transformation and a near-field computation based on the geometrical theory of diffraction is identical to the SNFGTD method originally used by F. Jensen and F. H. Larsen in 1977 [1]. The method was described in detail by $\mathrm{H}$. Bach in the report [2], the contents of which were presented at the NATO Advanced Study Institute in Norwich 1979. In the following years the method was further investigated by several researchers. Thus in 1981 an analysis of its accuracy as compared to physical optics (PO) and moment methods (MM) was performed by Bach, Frandsen, and Larsen. Some of their results are reported in [3], which also contains a description of the near-field calculation and the transformation techniques. Further analyses and applications were presented in [4] [6], and recently the method has been mentioned in the book by Dr. B. Westcott [7].

In their paper" Narasimhan and Christopher claim that "it is evident that the present method gives very good agreement with measured results" and later that the results can be made still more accurate by improving the near-field calculation and by increasing the number of spherical modes in the near field to farfield transformation. Although this has not been done, one can read in the abstract that "it is demonstrated that the technique proposed can predict the fields radiated by the reflector with greater accuracy by comparing the calculated results with the available measured results." Thus it is indicated to the reader that the SNFGTD method is superior to other methods in this respect.

In [3], which was brought to the attention of Narasimhan and Christopher by the referee of their paper, the curves shown in Fig. 1 were presented. These curves demonstrate that for a 20 wavelength reflector antenna fed by a dipole, practically identical $H$-plane patterns are obtained using SNFGTD, moment methods

Manuscript received March 19, 1984; revised July 9, 1984.

The author is with the Electromagnetics Institute, Technical University of Denmark, DK-2800, Lyngby, Denmark.

${ }^{1}$ M. S. Narasimhan and S. Christopher, IEEE Trans. Antennas Propagat., vol. AP-32, no. 1, pp. 13-19, Jan. 1984.

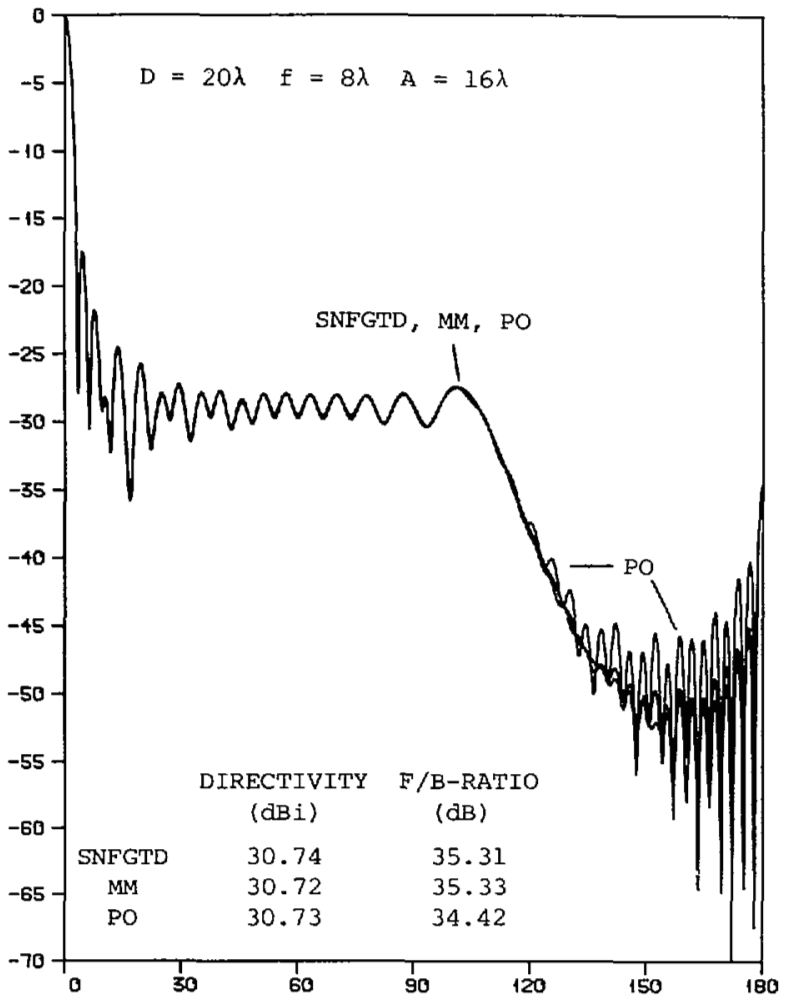

Fig. 1. Comparison of SNFGTD, mm, and PO for 20 wavelength reflector antenna.

and physical optics. The only significant differences occur in the region beyond $120^{\circ}$ where physical optics (not using far field GTD) differs from the other curves as could be expected. Thus, in 1981, it had been demonstrated that the method when used to calculate the $H$-plane pattern of a 20 wavelength antenna excited by a dipole yields results which are practically identical to those of other methods.

In order to restrict this communication as much as possible, I shall comment on only one of Narasimhan and Christopher's results, namely the $H$-plane radiation pattern shown in Fig. 2 of their paper. ${ }^{1}$ There they consider a focused parabolic reflector antenna with a diameter $D=10.65$ wavelengths, an $F / D$ ratio $=$ 0.25 and illuminated by a dipole, a configuration for which Afifi [8] made measurements in 1967. First of all, serious errors are observed in Narasimhan and Christopher's plot of the PO-GTD results of Koyoumjian [9]. For instance the level of the first sidelobe, as computed by Narasimhan and Christopher, is coincident with Afifi's measurements, but differs from physical optics by 3 $\mathrm{dB}$ approximately. This implies that physical optics predicts the level of the first sidelobe with an error of $3 \mathrm{~dB}$ approximately, a fact that I feel must be a big surprise to most antenna engineers. Furthermore, while measured and calculated results are almost coinciding on the center part of Kouyoumjian's curves they differ strongly in Narasimhan and Christopher's plot. These discrepancies may be due to bad plotting techniques, but in any circumstance it is not easy to accept the conclusions of Narasimhan and Christopher with regard to the accuracy of the SNFGTD method based on this background.

In Figs. 2(a) and 2(b) are presented patterns for the antenna in question computed at Technical University of Denmark by SNFGTD and physical optics supplemented by far field GTD in the 


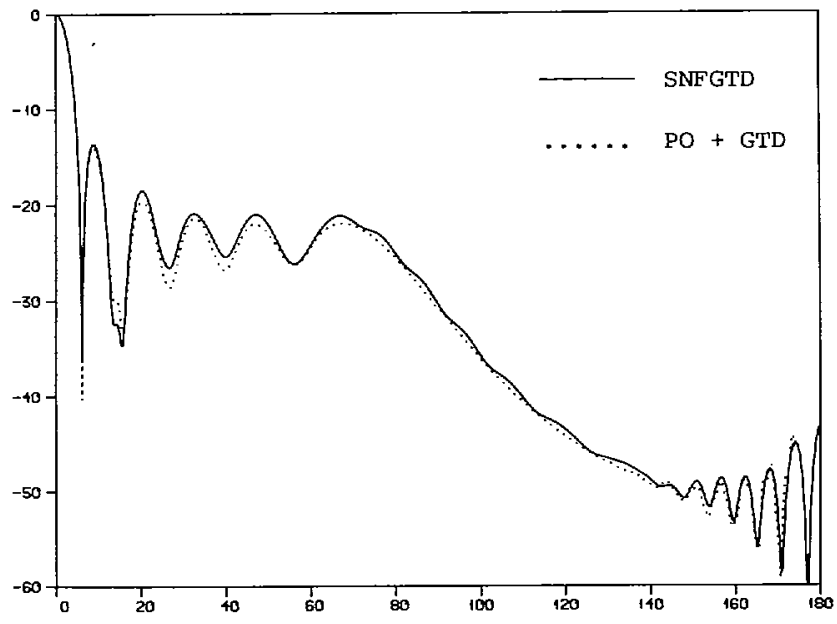

(a)

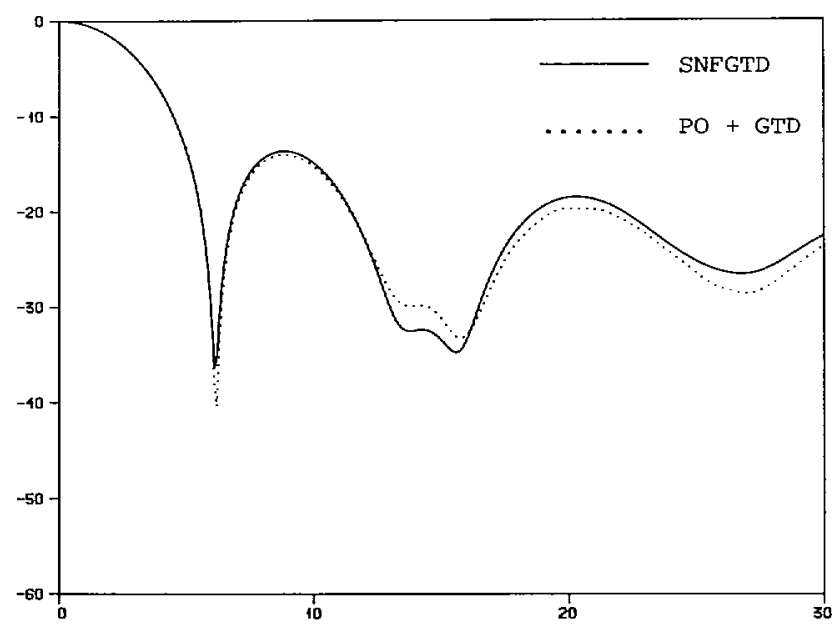

(b)

Fig. 2. (a) Comparison of SNFGTD, MM, second PO supplemented with GTD beyond $20^{\circ}$ for the Afifi antenna. (b) Comparison of SNFGTD, MM, second PO supplemented with GTD beyond $20^{\circ}$ for the Afifi antenna (expanded scale).

sidelobe region beyond $20^{\circ}$. From these it appears, contrary to the results of Narasimhan and Christopher, that the SNFGTD method, in this case and when used correctly, yields results that are almost identical to those obtained by physical optics. The small differences present are due to the small size of the reflector, since they are less pronounced for the larger reflector in Fig. 1. It is noted that the agreement between the two curves is obtained over the entire range from $0^{\circ}$ to $180^{\circ}$ and that the distortion of the pattern at $15^{\circ}$ is predicted by both methods. Thus it is the main conclusion of this communication that it is not possible to base any conclusions on the accuracy of the SNFGTD method on the results of Narasimhan and Christopher discussed here.

As a final remark I want to point out that the $H$-plane pattern is not very adequate for the purpose of assessing the accuracy of the method since the dipole excitation produces a strong spillover which obscures the details of the field from the reflector. Thus, in order to investigate the method in detail, antennas with a realistic horn excitation and other $F / D$-ratios should be used and computations of $E$-plane patterns and cross polar perform. ance should be carried out. Such results will be published in the near future by the author and his collaborators.

\section{REFERENCES}

[1] F. Jensen and F. H. Larsen, "Spherical near-field technique," in Antennas Propagat. Soc. Int. Symp. Dig., Univ. Stanford, 1977, pp. 378-381.

[2] H. Bach, "Field computations for reflector antennas by GTD," Electromagn. Inst., Tech. Univ. Denmark, 1979, NB 122.

[3] H. Bach, A. Frandsen, and F. H. Larsen, "Pattern prediction for a focused reflector antenna using GTD and near-field to far-field transformations," presented at Second Int. Conf. Antennas Propagat., Univ. York, Inst. Elec. Eng. Conf. Pub. 195, 1981, pp. 499-500.

[4] H. Bach, "Far-field computations for dual reflector antennas using GTD and near-field to far-field transformations," presented at Eighth Antenna Symp., QMC, Univ. London, 1982.

[5] H. Bach and H. H. Viskum, "Accurate near-field computations for axially defocused reflector antennas," presented at Eighth Antenna Symp., QMC, Univ. London, 1982.

[6] H. H. Viskum, "Pattern prediction for a defocused reflector antenna using GTD and near-field to far-field transformation," presented at Third Int. Conf. Antennas Propagat., Univ. East Anglia, Inst. Elec. Eng. Conf. Pub. 219, 1983, pp. 445-448.

[7] B. Westcott, Shaped Reflector Antenna Design. England: Research Studies, 1983.

[8] M. S. Afifi, "Radiation from the paraboloid of revolution," in Electromagnetic Wave Theory, pt. 2. New York: Pergamon, 1967, pp. 669-685.

[9] R. G. Kouyoumjian, "The geometrical theory of diffraction and its application," in Numerical and Asymptotic Techniques in Electromagnetics, R. Mittra Ed. New York: Springer-Verlag, 1975, ch. 6, p. 205.

\section{Authors' Reply by M. S. Narasimhan and S. Christopher ${ }^{2,3}$}

We would like to make the following observations on the comments made by H. Bach on our paper. ${ }^{1}$

It is surprising that Dr. Bach has not read our paper carefully. Our paper seeks to determine accurately both the near and far fields of a paraboloid (and this approach could be extended to any reflector antenna). We have also been able to provide an extrapolation formula for determination of the fields of the reflector in any radial direction ((13) and (14) in our paper $\left.{ }^{1}\right)$. The paper is merely an extension of the GTD analysis of the near fields of a reflector presented in our paper [10]. To our knowledge, we are the first to present this approach [10] in open literature. These contributions have not been made by Dr. Bach or anyone in open literature prior to our publication.

We do not think it is a sound argument to say that for the reflector antenna used by Afifi [8] the far-field computations based on physical optics approximation should agree completely in the sidelobe region starting from the first lobe with the more accurate computations based on a GTD-SME (spherical mode expansion) formulation proposed by us, since the aperture cdge illumination of the reflector under study is as low as $-6.021 \mathrm{~dB}$. This will certainly make the true current distribution on the reflector surface to be different from that obtained through the PO approximation. This will be acceptable by any student in electromagnetics.

If Dr. Bach is able to obtain identical far fields with PO approximation and hsi method of applying GTD and near-field/far-field transformation for the Afifi antenna, we suggest the editorial board go through the computer programs developed and used by us for calculating the far-field/near-field patterns of the Afifi reflector using PO as well as our GTD-SME technique and those

\footnotetext{
${ }^{2}$ Manuscript received August 21, 1984.

${ }^{3}$ The authors are with the Electromagnetics and Antennas Group, Centre for Systems and Devices, Indian Institute of Technology, Madras 600 036, India.
} 
of Dr. Bach and pass a verdict on the controversy. We do not think any other procedure will resolve this issue. We would also like to point out that the agreement between computations of the far fields based on PO and our GTD-SME technique in the sidelobe region strongly depends on 1) edge illumination for the dish, and 2) diameter of the dish.

Finally, we would like to add that we had computed the $E$-plane pattern also for the Afifi antenna but did not present it in order to keep the paper concise.

\section{REFERENCES}

[10] M. S. Narasimhan and K. M. Prasad, "GTD analysis of the near-field pattern of a prime-focus symmetric paraboloidal reflector antenna," IEEE Trans. Antennas Propagat., vol. AP-29, no. 6, pp. 959-961, Nov. 1981.

\section{Comments on Early Time SEM}

\author{
LEOPOLD B. FELSEN, FELLOW, IEEE
}

Recent studies of the singularity expansion method (SEM) for transient scattering from perfectly conducting targets have addressed the suitability of the resonance expansion for early times [1], [2]. Early observation times correspond to received signals from the partially illuminated scattering object, before the incident wavefront has excited it completely. It has been concluded that the conventional SEM expansion, with time-independent coupling coefficients derived from the complex frequency pole residues, is inadequate and must be augmented at early times by an entire function in the complex frequency plane. It has also been recognized that for low- $Q$ targets excited with broad-band illumination, most of the scattered energy resides in the early time interval. Thus, the entire function plays an essential role in constructing and interpreting the early time scattered response or, conversely, in using the early time response for the inverse problem of identification.

By its very nature, the early time response conveys local information pertaining to the excited portions of the scatterer. Global information, expressed by the resonances, becomes an effective descriptor only at later times, when the entire object has had a chance to contribute to the scattering process. Therefore, attempts to modify the resonance expansion at early times with time-varying coupling coefficients, while formally legitimate, still lead inherently to the "wrong" representation-local phenomena are synthesized poorly by superposition of many global events since heavy reliance is then placed on precise constructive and destructive interference.

Early time scattered fields have high frequency content. Therefore, they are organized naturally according to the hierarchy of reflected and diffracted fields of the geometrical theory of diffraction (GTD). In the time domain, the various rays of GTD identify distinct wavefront arrivals at the observer. These wave-

Manuscript received July 20, 1984. This work was supported in part by the Office of Naval Research under Contract N-00014-83-K-0214 and by the Joint Services Electronics Program under Contract F-49620-82-C-0084.

The author is with the Department of Electrical Engineering and Computer Science, Polytechnic Institute of New York, Route 110, Farmingdale, NY 11735 . front arrivals describe causal contributions due to surface reflection, diffraction at scattering centers, like edges, and complete circumnavigations (by creeping rays) of smooth convex shapes. Typical scattered field data for short pulse excitation clearly reveal the wavefront spikes at early times, as do numerical experiments conducted for various target shapes. In fact, time-dependent GTD applied to ultrasound is one of the more effective methods employed in nondestructive evaluation (NDE), where the goal is to find the location and shape of flaws and inclusions hidden in materials of various types [3]. While leading order GTD asymptotics is valid only for limited observation times after a wavefront arrival, more sophisticated spectral techniques [4] can be invoked to extend the range of validity to later times where lower frequencies begin to play a role. However, the early time interval is usually so short that simple asymptotics (based on WKB type approximations) should be adequate. The extended solutions become important when wavefront arrivals are counted even after the early time period has expired. This observation is relevant for the hybrid approach discussed below.

Extraction of a physical optics 'contribution from the resonance series conglomerate [1] is a step in the right direction to address the early time difficulties of SEM. But here, it should be noted that within the format of SEM, the frame of reference for axcitation of the scatterer is the location of the incident wavefront. This may cause some conceptual difficulties. On a smooth convex object, the incident wavefront excites the illuminated portion but in the geometrical shadow, surface points on the object are excited by the creeping wave. Thus, the creeping wavefront, establishes the first excitation on the shadowed side.

Therefore, it may be argued convincingly that observations of short pulse excited scattered fields during the early time interval, and even at moderate subsequent times, should be interpreted as multiple wavefront arrivals. Conversely, extraction of these arrivals from scattering data is likely to stabilize what remains. The later time response, which would involve too many wavefronts and also cause difficulty with low frequency tails of early arrivals, can be treated by resonances which represent the cumulative effect of all arrivals beyond those early ones that are kept intact. However, for low $Q$ targets excited by short pulses, the energy content in the late time signal may be so small as to render this portion insignificant. In any event, the hybrid wavefront-resonance formulation proposed recently [5] permits the smooth blending of these distinct constituents.

These ideas have been discussed elsewhere [6] and also in a forthcoming more detailed manuscript [7]. The wavefront interpretation of resonances, turn-on times, switch-on times and entire functions can remove much of the ambiguity that still besets these concepts within the SEM format. Ray fields corresponding to early times express the intrinsic entire function of SEM, which must be included. Retention of additional rays modifies the entire function and also the turn-on time, coupling coefficients and convergence rate of the SEM series. The hybrid approach shows that the coupling coefficients in the late time SEM series are proportional to the first ray field included in the collective ray field sum; the wavefront arrival time for that ray determines the turnon time of the SEM expansion and thereby provides a nice physical interpretation. Since higher order rays in a given category (for example, multiple diffracted rays between edges or creeping rays with multiple passes around a smooth object) are weakened with 